\title{
Weight Loss Trajectories and Psycho-Behavioral Predictors of Outcome of Primary and Reoperative Bariatric Surgery: A Two-Year Longitudinal Study
}

Ana Pinto-Bastos, ${ }^{1} \mathrm{PhD}$; Marta de Lourdes, ${ }^{1} \mathrm{MSc}$, Isabel Brandão, ${ }^{2} \mathrm{PhD}$, Paulo PP Machado, ${ }^{1} \mathrm{PhD}$, Eva M. Conceição, ${ }^{1 *} \mathrm{PhD}$

${ }^{1}$ University of Minho, School of Psychology, Campus Gualtar, 4710-057, Braga, Portugal

${ }^{2}$ Faculty of Medicine, University of Porto, Centro Hospitalar de São João, Porto, Portugal

\section{Correspondence to:}

*Eva M. Conceição; econceição@psi.uminho.pt ; School of psychology, University of Minho, Campus Gualtar, 4710-199, Braga, Portugal. Tlf: +351 253604 220; Fax: +351 253604224 .

Pinto-Bastos, A., de Lourdes, M., Brandão, I., Machado, P. P., \& Conceição, E. M. (2019). Weight loss trajectories and psychobehavioral predictors of outcome of primary and reoperative bariatric surgery: a 2-year longitudinal study. Surgery for Obesity and Related Diseases, 15(7), 1104-1112. https://doi.org/10.1016/j.soard.2019.04.018

(C)2019. This manuscript version is made available under the CC-BY-NC-ND 4.0 license http://creativecommons.org/licenses/by-nc-nd/4.0/

\section{Role of funding}

This study was partially conducted at Psychology Research Centre (PSI/01662), University of Minho, and supported by the Portuguese Foundation for Science and Technology and the Portuguese Ministry of Science, Technology and Higher Education through national funds, and co-financed by FEDER through COMPETE2020 under the PT2020 Partnership 
Agreement (POCI-01-0145-FEDER-007653), by the following grants to Eva Conceição (IF/01219/2014 and POCI-01-0145-FEDER-028209), and doctoral scholarship to Ana PintoBastos (SFRH/BD/104159/2014). The funding body had no role in the design, collection, analysis, and interpretation of data; the writing of the manuscript; or the decision to submit the manuscript for publication.

\section{Acknowledgements}

The authors which to acknowledge the AMTCO group (Avaliação Multidisciplinar do Tratamento Cirúrgico da Obesidade) at the Hospital of São João for conducting the multidisciplinary treatment as usual of the participants of this study.

Running title: PSYCHOLOGICAL ASPECTS OF REOPERATIVE SURGERY 
Title

\title{
Weight Loss Trajectories and Psycho-Behavioral Predictors of Outcome of Primary and Reoperative Bariatric Surgery: A Two-Year Longitudinal Study
}

\begin{abstract}
Background: Long-term behavioral and psychological aspects associated with weight outcomes following reoperative bariatric surgery have not been much investigated.
\end{abstract}

Objectives: This study sought (1) to identify differences in weight loss trajectories during the first 24 months in reoperative bariatric surgery (R-Group) and primary bariatric surgery (PGroup) and (2) to investigate pre- and post-surgery psycho-behavioral predictors of weight loss and weight regain for both groups.

Setting: Hospital center and university, Portugal.

Methods: This longitudinal study compared an R-Group ( $\mathrm{n}=157)$, and a P-Group $(\mathrm{n}=216)$. Patients were assessed at pre-surgery, and at 6, 12, 18 and 24 months post-surgery. Assessment included the EDE and Rep(eat) diagnostic interviews and a set of self-report measures assessing eating disorder symptomatology, grazing, depression, anxiety and impulsive behavior.

Results: P- and R-Groups presented a similar trajectory for the percentage of total weight loss $(\% \mathrm{TWL}) \quad(\beta=1.46, \mathrm{SE}=1.96 ; \mathrm{Wald} \chi 2=0.55, p=0.457) \quad$ and $\quad$ weight $\quad$ regain $(\beta=1.66, \mathrm{SE}=2.72 ; \mathrm{Wald} \chi 2=0.24, p=0.622)$. No significant pre-surgery predictors of weight loss and weight regain were found for the P-and R-Groups. Regarding post-surgery predictors, higher EDE-Q scores (Wald $\chi 2(1)=6.88, p=0.009)$ and grazing behavior (Wald $\chi 2(1)=8.30, p=0.004)$ were associated with less $\%$ TWL for both groups. Belonging to the P-Group emerged as a significant predictor of more weight loss (Wald $\chi 2(1)=7.25, p=0.007)$. Post-surgery anxiety predicted less \%TWL in R-Group (Wald $\chi 2(1)=3.89, p=0.043$ ). 
Considering weight regain, higher postoperative disordered eating (global EDE-Q) (Wald $\chi 2(1)=4.66, p=0.031)$ was associated with increased weight regain for P- and R-Groups. Conclusions: Problematic eating behaviors and psychological distress are significant predictors of poor weight outcomes for both groups.

Keywords: Reoperative bariatric surgery; trajectories of weight loss; predictors of outcome; longitudinal study. 


\section{Introduction}

Despite the significant weight loss induced by bariatric surgery, not all patients experience optimal long-term outcomes ${ }^{(\mathbf{1})}$ and some present long-term weight regain ${ }^{(\mathbf{1 , 2})}$. Predictors of poor outcomes among primary procedures have been widely studied although there are still inconclusive findings. For instance, the predictive value of pre- and post-surgical depression and anxiety in the short- and long-term results of weight loss after bariatric surgery remains controversial ${ }^{(3,4)}$. On the other hand, a growing body of literature have consistently shown that the post-surgery reemergence or development of dysfunctional eating patterns, such as patterns of uncontrolled, disordered eating or grazing ${ }^{(2,5)}$ and unhealthy food choices ${ }^{(6)}$, better predict poor outcomes.

With the increasing number of bariatric surgeries performed and consequent poor outcomes, the number of reoperative surgeries has been increasing ${ }^{(7)}$. Poor outcomes after primary surgery are multifactorial, involving insufficient weight loss, or weight regain, medical and surgical complications and poor adherence to prescribed lifestyle changes ${ }^{(8)}$. About 40.3$59 \%$ of the patients report inadequate weight loss or weight regain as the most frequent indication for the reoperation ${ }^{(8,9)}$. Although gastric banding is the procedure with the highest reoperation rate $(30.29 \%)^{(1)}$, it should be noted that patients who received primary gastric sleeve gastrectomy $^{(10)}$ or gastric bypass also undergo secondary surgeries ${ }^{(11)}$.

Despite inducing significant weight loss, these secondary surgical procedures are associated with increased complications and higher mortality rates ${ }^{(12)}$. Unfortunately, there are still few long-term reports on the behavioral and weight impact of these surgeries, with most studies reporting on less than two-year after surgery (e.g. $\left.{ }^{(6)}\right)$.

Long-term behavioral and psychological factors associated with poor outcomes following reoperative bariatric surgery have not been much documented. Recent research compared psycho-behavioral outcomes of reoperative and primary surgery patients before surgery and 6 
months after surgery ${ }^{(9,13)}$. Findings from these studies suggest that reoperative patients tend to engage in problematic eating behaviors more than primary surgery patients, particularly in preoperative binge-eating episodes ${ }^{(9)}$, and grazing behavior at 6 months postsurgery ${ }^{(13)}$. Despite the identified differences, both groups presented several similarities with regards to eating behavior and related psychological aspects at pre- ${ }^{(9)}$ and 6 months post-surgery ${ }^{(13)}$. Kafri et al. ${ }^{(6)}$ found similar results in a study with a longer follow-up (18 months), pointing out that patients undergoing a secondary surgery reported more non-normative eating patterns and less healthy food selection than primary surgery patients. In addition to eating behaviors, Pinto-Bastos et al. ${ }^{(13)}$ reported that patients undergoing reoperative surgeries presented greater post-surgery anxiety which was associated with less weight lost in this group.

Given the variability of results reported in the literature regarding weight outcomes observed in patients undergoing reoperative surgery ${ }^{(6,14,15)}$, it is important to clarify whether these patients lose less weight than primary patients. Reoperative patients - who have already experienced poor primary outcomes - may represent a high-risk group, with more problematic eating behaviors and higher general psychopathology levels, which may compromise the results of the secondary surgery, eventually leading patients to a third surgery.

Researchers have widely focused their attention on primary bariatric surgery, however it is central to investigate and understand whether reoperative patients have the same factors and outcomes as patients undergoing primary surgery. Our study intends to provide data on this understudied population. To the best of our knowledge, no study has yet investigated weight loss or weight gain trajectories in reoperative bariatric surgery. Identifying the trajectories of weight loss and weight regain in secondary surgery could be useful in detecting relevant moments for the identification of the psychological and behavioral aspects associated with poor weight outcomes, contrasting these trajectories with those of primary patients. Additionally, we sought to investigate pre- and post-surgery psycho-behavioral predictors of weight loss and 
weight regain for different trajectories for reoperative bariatric surgery (R-Group) and primary bariatric surgery (P-Group).

\section{Materials and Methods}

Procedure

This is a longitudinal study conducted in a central hospital in the north of Portugal, that assessed consecutive patients undergoing primary and reoperative bariatric surgery at presurgery assessment, and 6,12,18, and 24 months after surgery. All patients admitted to bariatric surgery from April 2014 to April 2017 were considered eligible for the study. All bariatric surgery candidates were evaluated the month prior to surgery in the day of their medical appointment. The follow-up assessments were conducted after their medical appointments at the hospital.

Exclusion criteria included pregnancy after surgery, severe cognitive compromise that limited patients' autonomy, acute presentation of psychiatric conditions with severe impairment of global functioning, major active psychiatric disorder other than an eating disorder as primary diagnosis, and undergoing ketogenic or liquid diets during the months before surgery.

Both the University and the Hospital's Institutional Review Boards approved this research. Patients were informed about the confidentiality of the data collected and signed an informed consent form accepting to participate in the study.

\section{Participants}

In total, 395 patients undergoing bariatric surgery were eligible for this study, but four patients did not accept participation and eighteen completed only baseline assessment, refusing to continue their participation. The baseline assessment included 216 patients undergoing primary surgery and 157 undergoing reoperative surgery. Figure 1 shows the flowchart of the 
data collection. All reoperative patients in the study received adjustable gastric band as their first bariatric surgery.

***Insert figure 1 here $* * *$

\section{Measures}

Weight outcomes: Height, pre-surgery, nadir weight (lowest since surgery), and postsurgery weights were acquired from medical charts. Regarding R-Group, the weight preprimary surgery was self-reported by patients. The different weight related variables were computed as follows: Body mass index (BMI): weight/(height $\left.{ }^{2}\right)$; Percentage of total weight loss $(\% T W L):(($ weight pre-surgery-weight post-surgery)/weight pre-surgery)*100; and weight regain was quantified as Percentage of Maximum Weight Lost (\%MWL): (post-nadir weight - nadir weight)/(pre-surgery weight - nadir weight)*100(16).

Face-to-face clinical interview: A semi-structured clinical interview was used to assess socio-demographic variables and clinical features. To assess and stipulate problematic and dysfunctional eating behaviors, the diagnostic items of the Eating Disorders Examination ${ }^{(17)}$ was used. This face-to-face interview assessed objective binge eating (OBE) episodes characterized by episodes of eating an objectively large amount of food with a feeling of loss of control and subjective binge eating (SBE) episodes considered when eating small/modest amounts of food with loss of control perceived as excessive by the respondent. The Rep(eat) interview $^{(18)}$ was used to assess the grazing behavior, generally defined as eating repetitively small amounts of food in an unplanned manner.

\section{Self-report measures:}


Eating Disorder Examination-Questionnaire, EDE- $Q^{(19,20)}$ : This self-report measure was used to assess eating disorder psychopathology and associated features. This 28-item questionnaire generates four subscale scores (restraint, eating concern, shape concern, and weight concern), as well as a global score.

Depression Anxiety Stress Scale - DASS-21 (21,22): The Portuguese version ${ }^{(22)}$ of this 21item measure evaluates depression, anxiety and stress symptoms.

Urgency, Premeditation, Perseverance, and Sensation Seeking scales - UPPS ${ }^{(23)}$ : This instrument was developed with the purpose of evaluating impulsivity behaviors. Only the Negative Urgency subscale was used in this study to evaluate impulsivity when accompanied by negative emotions.

Repetitive eating questionnaire - Rep(eat)- $Q^{(24)}$ : This is a 12 -item measure validated in Portuguese that assesses a grazing-type eating pattern and generates two subscales: compulsive grazing and repetitive eating, as well as a total score.

Three Factors Eating Questionnaire-Revised 21 - TFEQ-R21 $1^{(25,26)}$ : This is a 21 -item measure that generates three subscales: uncontrolled eating; cognitive restraint and emotional eating.

\section{Statistical Analysis}

Statistical power was computed using GPower software 3.1, given the $\alpha$, sample size and effect size, for F-tests considering a statistical power of $80 \%$ with a moderated effect size of 0.3 , assuming an $\alpha$-error of $5 \%$, resulting in a sample size of 370 .

Generalized estimating equations (GEE) with growth curve analyses were used to investigate trajectories over time in percentage of total weight loss $(\% \mathrm{TWL})$ and percentage of maximum weight lost (\%MWL) for $\mathrm{P}$ - and R-Groups. GEE allows for the analysis of participants with missing data at some, but not all, time points. The GEEs tested hypotheses 
about $\%$ TWL and \%MWL changes, time course, and group $\mathrm{x}$ time interactions, with linear, quadratic, and cubic components. Growth parameters included time in months following surgery (linear slope), time quadratic (quadratic slope) and time cubic (cubic slope). The prediction curves for the models tested were plotted through the following equations:

$\%$ TWLti $=\beta$ int $+\beta$ Group $+\beta$ lin $($ time $)+\beta$ qua $(\text { time })^{2}+\beta$ cu $(\text { time })^{3}+\beta$ Groupxlin(time $)+\beta$ Gro upxqua(time $)^{2}+\beta$ Groupxcu(time $)^{3}$, where $\%$ TWLti=percentage of total weight loss at each assessment time; int=Intercept value; Group=P-Group or R-Group; lin=linear slope parameter; qua $=$ quadratic slope parameter; $\mathrm{cu}=$ cubic slope parameter.

$\% \mathrm{MWL} \mathfrak{t}_{\mathrm{i}}=\beta_{\text {int }}+\beta_{\text {Group }}+\beta_{\text {lin }}($ time $)+\beta_{\text {qua }}(\text { time })^{2}+\beta$ cu(time $)^{3}+\beta_{\text {Groupxlin }}($ time $)+\beta G_{\text {roupxqua }}($ ti me $)^{2}+\beta_{\text {Groupxcu }}(\text { time })^{3}$, where $\% \mathrm{MWLt}_{\mathrm{i}}=$ percentage of maximum weight lost at each assessment time; int=Intercept value; Group=P-Group or R-Group; lin=linear slope parameter; $q u a=$ quadratic slope parameter; $c u=$ cubic slope parameter.

The type of surgery was tested as co-variant in all models included.

In order to investigate if pre- and post-operative factors predict long-term weight outcomes following surgery for both groups, pre- and post-operative variables were tested. Four models with the variable group were tested that included pre- and post-surgery predictors for $\%$ TWL and \%MWL, separately. Generalized linear models (GLM) with linear response for $\%$ TWL with gamma distribution and log link for weight regain were tested to identify predictors of percentage of \%TWL and \%MWL, respectively.

Analyses were performed using the IBM SPSS ${ }^{\circ}$ statistics V24 software and $p$ values under .05 were considered statistically significant. 


\section{Results}

\section{Characterization of the Sample}

In the R-Group, $42.7 \%$ of patients $(n=67)$ mentioned weight regain/poor weight loss as the reason for reoperative surgery, while $33.8 \%(n=53)$ referred to anatomical and medical complications, and $23.6 \%(\mathrm{n}=37)$ mentioned both. No significant differences between $\mathrm{P}$ - and R-Groups were found for sociodemographic variables except for age and gender. Compared to patients in the P-Group, patients undergoing reoperative surgery were older (mean of 4.37 years older) and included less male participants than the P-Group of patients ( $12 \%$ vs $5.7 \%)$. Table 1 presents the detailed sociodemographic characterization of this sample.

***Insert Table 1 about here $* * *$

\section{Trajectories of \% total weight loss after bariatric surgery in P-Group and R-Group}

P- and R-Group presented a similar trajectory regarding \%TWL across time since no significant interaction effects were found regarding linear $(\beta=3.63, \mathrm{SE}=2.89 ;$ Wald $\chi 2=1.57, p=0.210)$, quadratic $(\beta=-0.97, \mathrm{SE}=1.11 ;$ Wald $\chi 2=0.76, p=0.383)$ and cubic $(\beta=0.08, \mathrm{SE}=0.13 ;$ Wald $\chi 2=0.35, p=0.554)$ growth parameters, suggesting that both groups showed a similar evolution across time $(\beta=1.46, \mathrm{SE}=1.96 ; \mathrm{Wald} \chi 2=0.55, p=0.457)$. The model showed a significant main effect for time in the linear slope $(\beta=17.08, \mathrm{SE}=2.28$; Wald $\chi 2=170.47, p=0.000)$ suggesting an initial significant increase in the \%TWL for both groups. A significant main effect with quadratic slope ( $\beta=-$ 4.70, $\mathrm{SE}=0.89 ;$ Wald $\chi 2=86.53, p=0.000)$ suggested a similar trend for both groups as time elapsed with a deceleration of the rate of \%TWL between 12 and 18 months after surgery 
(Figure 2). The significant main effect with cubic slope $(\beta=0.40, \mathrm{SE}=1.96 ; \mathrm{Wald} \chi 2=45.47, p=0.000)$ depicted a slight decrease in weight loss outcomes for both groups between 18 and 24 months.

\section{***Insert Figure 2 about here $* * *$}

\section{Trajectories of \% of maximum weight lost after bariatric surgery in P-Group and R-Group}

GEE models showed non-significant interaction effects between time and groups, regarding linear $\quad(\beta=1.38, \mathrm{SE}=5.63$; Wald $\chi 2=0.06, p=0.806), \quad$ quadratic $\quad(\beta=-$ $1.03, \mathrm{SE}=2.82 ; \mathrm{Wald} \chi 2=0.13, p=0.715)$ and cubic $(\beta=0.15, \mathrm{SE}=0.42 ; \mathrm{Wald} \chi 2=0.13, p=0.718)$ growth parameters suggesting that $\mathrm{P}$ - and $\mathrm{R}$ - Groups present similar trajectories of $\% \mathrm{MWL}(\beta=-$ $0.50, \mathrm{SE}=3.26$; Wald $\chi 2=0.68, p=0.410)$. A non-significant main effect for time with the linear slope $(\beta=-3.56, \mathrm{SE}=4.42 ; \mathrm{Wald} \chi 2=1.04, p=0.308)$ was found, as well as non-significant main effect with quadratic and cubic slopes $(\beta=2.15, \mathrm{SE}=2.19$; Wald $\chi 2=1.34, \quad p=0.248), \quad(\beta=-$ $0.17, \mathrm{SE}=0.32 ; \mathrm{Wald} \chi 2=0.22, p=0.637$ ), respectively (Figure 3 ), suggesting that both groups regain identical weight over time.

\section{***Insert Figure 3 about here $* * *$}

\section{Predictors of weight loss and weight regain after bariatric surgery in P-Group and R-Group}

This study also intended to investigate predictors of the \%TWL and WR after 24 months surgery for both groups. Predictors tested included the pre- and 24 months post-surgery scores on the global EDE-Q and subscales, depression, anxiety and stress subscales, negative urgency (UPPS-P), total Rep(eat)-Q and subscales, TFEQ-R21 subscales (uncontrolled eating, cognitive restraint and emotional eating), and frequency of different problematic eating behaviors (the 
number of days in the previous month with $\mathrm{OBE}, \mathrm{SBE}$ and grazing behavior). These variables were tested as main effect and as interaction effect with the group variable (variable $\mathrm{x}$ group (P-Group/R-Group)). This approach was used to identify specific predictors of long-term weight loss and weight regain $(\% \mathrm{MWL})$ in each group.

Investigating pre-surgery predictors of $\% \mathrm{TWL}$, no significant predictors were found for P-group nor R-Group (likelihood ratio $\chi 2=12.63, p=0.125$ ). With regards to the post-surgery predictors of \%TWL after 24 months surgery, we found that higher disordered eating (global EDE-Q) $($ Wald $\chi 2(1)=6.88, p=0.009)$ and grazing behavior $(\operatorname{Rep}($ eat $)-Q) \quad($ Wald $\chi 2(1)=8.30$, $p=0.004)$ were associated with less $\%$ TWL for P-Group and R-Group. Belonging to the P-group emerged as significant predictor of more weight loss (Wald $\chi 2(1)=7.25, p=0.007)$. The interaction effect between anxiety and group (Wald $\chi 2=3.89, p=0.043$ ) suggested that anxiety is associated with lower the \%TWL for the reoperative group of patients.

In investigating predictors of weight regain (\%MWL), no significant predictors presurgery (likelihood ratio $\chi 2=9.90, p=0.702$ ) were found for any of the groups. Considering postsurgery predictors, higher disordered eating (global EDE-Q) (Wald $\chi 2(1)=7.78, p=0.005)$ was associated with increased weight regain for P-and R-Groups. No predictor of interaction effect was found.

Patients who completed follow-up versus and those who were lost in this study did not differ in any of the relevant aspects: trajectories of $\%$ TWL and \%MWL, pre- and post-surgery predictors of \%TWL and \%MWL (data not -shown but available upon request). 


\section{Discussion}

This study sought to bring evidence for the behavioral and psychological aspects and weight outcomes in patients undergoing reoperative bariatric surgery, and to compare this population with patients undergoing a primary surgery.

We aimed at identifying different trajectories of weight outcomes during the 24 months following bariatric surgery in both patients undergoing primary and secondary surgery. Both groups presented similar trajectories regarding \%TWL across time. Although the literature shows mixed results, our results advocate that reoperative patients achieve results comparable to those of patients undergoing a primary bariatric procedure ${ }^{(14,27)}$. P- and R-Groups presented a slight trend for deceleration in results of weight loss between 12 and 18 months after surgery. Our findings add support to the fact that, similarly to primary surgery, the majority of the weight loss occurs within the first year after bariatric surgery ${ }^{(11,15)}$. Our data showed a slight decrease in weight loss outcomes for both groups between 18 and 24 months. Nevertheless, some studies suggests that weight loss stabilization is observed between 18 and 24 months post-surgery in both groups ${ }^{(28)}$.

According to King et al. ${ }^{(16)}$, we quantified weight regain as \%MWL. As for $\% \mathrm{TWL}$, trajectories of \%MWL following surgery were not distinct for P-Group and R-Group up until a 24 months follow-up, which brings additional light for early preventive strategies for weight regain in both groups. However, it is known that in primary bariatric surgery, weight regain appears within two years and five after surgery ${ }^{(29,30)}$. Thus, longer-term assessments are needed to investigate if the trajectories of weight loss and weight regain are sustained over time or if the results tend to deteriorate, particularly in reoperative patients.

Finally, we intended to investigate pre- and post-surgery psycho-behavioral predictors of weight loss and weight regain for different trajectories for both P- and R-Groups. No significant pre-predictors of $\%$ of weight loss and $\%$ of maximum weight lost were found for either group. 
We found that belonging to the P-Group was a predictor of more weight loss. Although results' variability seems to be characteristic of the literature related to this question, some studies have shown that patients undergoing reoperative bariatric surgery lose less weight than primary surgery patients ${ }^{(6,15)}$. Some reasons may explain inconsistent results in the literature. In our study, it should be noted that in the P-Group, the patients were younger and had more male participants than R-Group, which may have an impact on some extent in the weight loss results. Another possible reason is the our sample only included reoperative patients who had performed the gastric band as first surgical procedure, and the literature has shown that procedure purely restrictive lose more weight than patients undergoing prior malabsorptive procedures $^{(31)}$. Both groups shared some of the same predictors of poor weight loss and weight regain outcomes. More postoperative disordered eating (global EDE-Q) and grazing behavior (Rep(eat)-Q) were associated with less \%TWL for the P- and R-Group at the 24 months followup. Past research has emphasized that post-surgery behavioral and psychological variables best predict weight outcomes, in detriment of pre-surgical variables ${ }^{(2)}$. In fact, previous studies found similar outcomes concerning post-surgery problematic eating behaviors and grazing in worse long-term weight loss in primary bariatric surgery ${ }^{(18,32,33)}$ and short-term weight loss in reoperative patients ${ }^{(13)}$. Colles et al. ${ }^{(32)}$ pointed out that the overlap between grazing and uncontrolled eating postoperatively predicted lesser weight loss in patients undergoing primary surgery. Regarding the predictors of weight regain $(\% \mathrm{MWL})$, we found that higher postoperative disordered eating (global EDE-Q) was associated with increased weight regain for P- and R-Groups. This finding corroborates existing research since problematic eating behaviors have been related with bariatric surgery outcomes, particularly weight regain(34).

Despite the identified common predictors, we found different predictors of weight loss between P- and R-Groups, particularly anxiety. The groups were distinguished for post-surgery anxiety, since reoperative patients presented more anxiety that predicts less weight loss at two 
years after surgery. This result had previously been observed in a recent study with the same sample in earlier stages of reoperative surgery, namely at 6 months after surgery ${ }^{(13)}$. Patients with anxiety and mood symptomatology may be more susceptible to adopt dysfunctional eating behaviors, affecting compliance with medical prescriptions ${ }^{(35,36)}$ and therefore the weight outcomes. Besides that, another possibility for this outcome, may be the fact that these patients undergoing a second surgery, having failed in the results of weight in first surgery, may be more focused on losing weight. This greater focus may trigger more anxious symptomatology, which may therefore have a negative impact on weight loss. Thus, it would be relevant to investigate in future research whether patients undergoing reoperation due to weight regain / poor weight loss related to psychological and behavioral issues may reappear in the second surgery and how they may affect outcomes.

The similarities and differences observed in behavioral and psychological factors in primary and reoperative patients add to and highlight the implications for post-surgical consulting and treatment. In this sense, it is important to detect the risk factors associated with poor weight outcome in this specific population, suggesting that early detection may be favorable for the reoperative patients. These findings show evidence for monitoring individuals with anxiety, as well as disordered eating, for poor weight loss after surgery.

Although the data did not show differences between groups in the weight trajectories, together, these results propose that the reemergence or development of problematic eating behaviors, greater psychological distress and disordered eating psychopathology seem to be risk factors for poorer weight outcomes in primary and reoperative bariatric patients ${ }^{(6,13)}$. The predictive value of eating features and psychological variables, and its strong association with weight outcomes after bariatric surgery in both groups, particularly at long-term, highlight the importance of systematic and continuous monitoring from initial stages ${ }^{(13)}$ to long-term follow$u^{(2)}$. Both groups seem to need psycho-behavioral and support group interventions to optimize 
outcomes, in order to maintain the long-term resolution of the comorbidities associated with obesity and, ultimately, preventing significant weight regain that may justify more invasive strategies, such as secondary and tertiary surgery. Research has been emphasizing the role of post-surgery interventions in the promotion of post-surgery weight loss and weight control by means of increased adherence to recommendations, motivation, and increased awareness of eating patterns and better psychosocial functioning ${ }^{(37)}$.

The major strengths of this study include the large sample size of obese participants, the long-term follow-up period, longitudinal design, the fact that anthropometric data were retrieved from medical charts instead of self-reported, and the use of validated interviews to assess the different types of problematic eating. Despite the strengths of this study, it is important to acknowledge some limitations. An important limitation of this study was the missing data in all assessment moments, translating into patient's loss of follow-up: in spite of the several attempts made to reschedule their visits or to collect data via phone, patients did not show up to the reschedule session or did not answer the phone. Others patients changed their telephone number, leaving us with no access to the new contact. Also, the study was performed only in a specific hospital in the north of Portugal, which may limit the generalization of the findings to different national and international bariatric samples. Other limitation concerning the generalization of results relates to the fact that the reoperative group consisted only of patients receiving secondary surgery after a primary gastric band. Finally, longer follow-up times is needed to understand these results are sustained over time or if tend to deteriorate. 


\section{Conclusion}

Patients undergoing reoperative and primary surgery presented a similar trajectory for the $\%$ TWL and weight regain during the 24 months. Problematic eating behaviors and psychological distress are significant predictors of poor weight outcomes for both groups.

These data suggest that primary and reoperative patients may be at risk of poorer longterm weight outcomes and may need specific interventions focused on post-surgery psychological and behavioral aspects for this seems to be an important strategy for optimizing long-term outcomes, and consequently prevent a secondary or tertiary surgery.

\section{Disclosure Statement}

None of the authors have any conflict of interest associated with this work to declare. 


\section{References}

1. Courcoulas AP, King WC, Belle SH, Berk P, Flum DR, Garcia L, et al. Seven-Year Weight Trajectories and Health Outcomes in the Longitudinal Assessment of Bariatric Surgery (LABS) Study. JAMA Surg. 2017;153(5):427-34. doi:10.1001/jamasurg.2017.5025

2. Conceição E, Mitchell JE, Pinto-Bastos A, Arrojado F, Brandão I, Machado PPP. Stability of problematic eating behaviors and weight loss trajectories after bariatric surgery: a longitudinal observational study. Surg Obes Relat Dis. 2017;13(6):1063-70. doi:10.1016/j.soard.2016.12.006

3. de Zwaan M, Enderle J, Wagner S, Mühlhans B, Ditzen B, Gefeller O, et al. Anxiety and depression in bariatric surgery patients: A prospective, follow-up study using structured clinical interviews. J Affect Disord. 2011;133(1-2):61-8. doi:10.1016/j.jad.2011.03.025

4. Hayden MJ, Murphy KD, Brown WA, O’Brien PE. Axis I disorders in adjustable gastric band patients: The relationship between psychopathology and weight loss. Obes Surg. 2014;24(9):1469-75. doi: 10.1007/s11695-014-1207-0

5. Meany G, Conceição E, Mitchell JE. Binge eating, binge eating disorder and loss of control eating: Effects on weight outcomes after bariatric surgery. Eur Eat Disord Rev. 2014;22(2):87-91. doi:10.1002/erv.2273

6. Kafri N, Valfer R, Nativ O, Shiloni E, Hazzan D. Behavioral outcomes following laparoscopic sleeve gastrectomy performed after failed laparoscopic adjustable gastric banding. Obes Surg. 2013;23(3):346-52. doi:10.1007/s11695-012-0794-X

7. Hii MW, Lake AC, Kenfield C, Hopkins GH. Laparoscopic conversion of failed gastric banding to Roux-en-Y gastric bypass. Short-term follow-up and technical considerations. Obes Surg. 2012;22(7):1022-8. doi:10.1007/s11695-012-0594-3 
8. Deylgat B, Hondt MD, Pottel H, Vansteenkiste F, Rooy F Van, Devriendt D. Indications, safety, and feasibility of conversion of failed bariatric surgery to Roux-enY gastric bypass : a retrospective comparative study with primary laparoscopic Rouxen-Y gastric bypass. Surg Endosc. 2012;26(7):1997-2002. doi:10.1007/s00464-0112140-0

9. Conceição E, Pinto-Bastos A, de Lourdes M, Brandão I, Teixeira C, Machado PP. Psychological, behavioral and weight-related aspects of patients undergoing reoperative bariatric surgery after gastric band: Comparison with primary surgery patients. Surg Obes Relat Dis. 2018;14(5):603-10. doi:10.1016/j.soard.2018.02.011

10. Homan J, Betzel B, Aarts EO, Van Laarhoven KJHM, Janssen IMC, Berends FJ. Secondary surgery after sleeve gastrectomy: Roux-en-Y gastric bypass or biliopancreatic diversion with duodenal switch. Surg Obes Relat Dis. 2015;11(4):7717. doi:10.1016/j.soard.2014.09.029

11. Gobble RM, Parikh MS, Greives MR, Ren CJ, Fielding GA. Gastric banding as a salvage procedure for patients with weight loss failure after Roux-en-Y gastric bypass. Surg Endosc. 2008;22(4):1019-22. doi:10.1007/s00464-007-9609-x

12. Himpens J, Coromina L, Verbrugghe A, Cadière G-B. Outcomes of revisional procedures for insufficient weight loss or weight regain after Roux-en-Y gastric bypass. Obes Surg. 2012;22(11):1746-54. doi:10.1007/s11695-012-0728-7

13. Pinto-Bastos A, Conceição E, de Lourdes M, Arrojado F, Brandão I, Machado PPP. Psychological and Behavioral Aspects of Primary and Reoperative Surgery: a 6-Month Longitudinal Study. Obes Surg. 2018; 28(12), 3984-91. doi:10.1007/s11695-018-34520

14. Jennings N a., Boyle M, Mahawar K, Balupuri S, Small PK. Revisional laparoscopic Roux-en-Y gastric bypass following failed laparoscopic adjustable gastric banding. 
Obes Surg. 2013;23(7):947-52. doi:10.1007/s11695-013-0888-0

15. Zingg U, McQuinn A, Divalentino D, Kinsey-Trotman S, Game P, Watson D.

Revisional vs. primary Roux-en-Y gastric bypass-a case-matched analysis: Less weight loss in revisions. Obes Surg. 2010;20(12):1627-32. doi:10.1007/s11695-010-0214-Z

16. King WC, Hinerman AS, Belle SH, Wahed AS, Courcoulas AP. Comparison of the Performance of Common Measures of Weight Regain after Bariatric Surgery for Association with Clinical Outcomes. JAMA 2018;320(15):1560-9. doi:10.1001/jama.2018.14433

17. Fairburn CG, Cooper Z, O’Connor M. Eating disorder examination. In: Fairburn CG (ed). Cognitive behavior therapy and eating disorders. New York: Guilford Press; 2008.

18. Conceição EM, Mitchell JE, Engel SG, Machado PPP, Lancaster K, Wonderlich SA. What is "grazing"? Reviewing its definition, frequency, clinical characteristics, and impact on bariatric surgery outcomes, and proposing a standardized definition. Surg Obes Relat Dis. 2014;10(5):973-82. doi:10.1016/j.soard.2014.05.002

19. Fairburn CG, Beglin SJ. Assessment of eating disorders: Interview or self-report questionnaire? Int J Eat Disord. 1994;16(4):363-370. doi:10.1002/1098108X(199412)16:4<363::AID-EAT2260160405>3.0.CO;2-\#

20. Machado P, Martins C, Vaz A, Conceição E, Bastos AP, Gonçalves S. Eating Disorder Examination Questionnaire (EDE-Q): Psychometric Properties and Norms for the Portuguese Population. Eur Eat Disord Rev. 2014; 22(6):448-53. doi:10.1002/erv.2318

21. Lovibond SH, Lovibond PF. Manual for the depression, anxiety, stress scales. Sydney: Psychology Foundation; 1995.

22. Pais-Ribeiro JL, Honrado A, Leal I. Contribuição Para O Estudo Da Adaptação Portuguesa Das Escalas De Ansiedade, Depressão e Stress (EADS) De 21 Itens De 
Lovibond E Lovibond. Psicol Saúde Doenças. 2004;5(2):229-39.

doi:10.1080/13548500500524088

23. Whiteside SP, Lynam DR, Miller JD, Reynolds SK. Validation of the UPPS Impulsive Behavior Scale: A four factor model of impulsivity. Eur J Pers 2005;574(19):559-74. doi:10.1002/per.556

24. Conceição EM, Mitchell JE, Machado PPP, Vaz AR, Pinto-Bastos A, Ramalho S, et al. Repetitive eating questionnaire [Rep(eat)-Q]: Enlightening the concept of grazing and psychometric properties in a Portuguese sample. Appetite. 2017;117:351-8. doi:10.1016/j.appet.2017.07.012

25. Duarte P, Palmeira L, Pinto-Gouveia P. The Three-Factor Eating Questionnaire-R21: A confirmatory factor analysis in a Portuguese sample. Eat Weight Disord. 2018. doi:10.1007/s40519-018-0561-7

26. Cappelleri JC, Bushmakin AG, Gerber RA, Leidy NK, Sexton CC, Karlsson J. Psychometric analysis of the Three-Factor Eating Questionnaire-R21: results from a large diverse sample of obese and non-obese participants. Int J Obes. 2009;33(6):61120. doi:10.1038/ijo.2009.74

27. Topart P, Becouarn G, Ritz P. One-year weight loss after primary or revisional Rouxen-Y gastric bypass for failed adjustable gastric banding. Surg Obes Relat Dis. 2009;5(4):459-62. doi:10.1016/j.soard.2008.08.008

28. O’Brien PE, Dixon JB, Brown W. Obesity is a surgical disease: Overview of obesity and bariatric surgery. ANZ J Surg. 2004;74(4):200-4. doi:10.1111/j.14452197.2004.03014.x

29. Magro DO, Geloneze B, Delfini R, Pareja BC, Callejas F, Pareja JC. Long-term weight regain after gastric bypass: A 5-year prospective study. Obes Surg. 2008;18(6):648-51. doi:10.1007/s11695-007-9265-1 
30. Langer FB, Prager G, Poglitsch M, Kefurt R, Shakeri-Leidenmühler S, Ludvik B, et al. Weight loss and weight regain - 5-year follow-up for circular- vs. linear-stapled gastrojejunostomy in laparoscopic roux-en-Y gastric bypass. Obes Surg. 2013;23(6):776-81. doi:10.1007/s11695-013-0892-4

31. Brolin RE, Cody RP. Weight loss outcome of revisional bariatric operations varies according to the primary procedure. Ann Surg. 2008;248(2):227-32. doi:10.1097/SLA.0b013e3181820cdf

32. Colles SL, Dixon JB, O’Brien PE. Grazing and loss of control related to eating: two high-risk factors following bariatric surgery. Obesity. 2008;16(3):615-22. doi:10.1038/oby.2007.101

33. Pinto-Bastos A, Conceição EM, Machado PPP. Reoperative Bariatric Surgery: a Systematic Review of the Reasons for Surgery, Medical and Weight Loss Outcomes, Relevant Behavioral Factors. Obes Surg. 2017;27(10):2707-15. doi:10.1007/s11695$017-2855-7$

34. Conceição EM, Utzinger LM, Pisetsky EM. Eating disorders and problematic eating behaviours before and after bariatric surgery: Characterization, assessment and association with treatment outcomes. Eur Eat Disord Rev. 2015;23(6):417-25. doi:10.1002/erv.2397

35. Kalarchian MA, Marcus MD, Levine MD, Soulakova JN, Courcoulas AP, Wisinski MSC. Relationship of psychiatric disorders to 6-month outcomes after gastric bypass. Surg Obes Relat Dis. 2008;4(4):544-9. doi:10.1016/j.soard.2008.03.003

36. Legenbauer T, Petrak F, De Zwaan M, Herpertz S. Influence of depressive and eating disorders on short- and long-term course of weight after surgical and nonsurgical weight loss treatment. Compr Psychiatry. 2011;52(3):301-11. doi:10.1016/j.comppsych.2010.06.012 
37. Rudolph A, Hilbert A. Post-operative behavioural management in bariatric surgery: A systematic review and meta-analysis of randomized controlled trials. Obes Rev. 2013;14(4):292-302. doi:10.1111/obr.12013 


\section{Legends:}

Table 1- Characterization of the sample: socio-demographic

Figure 1 - Flow diagram of data collection

Note. *Patients who were lost at specific follow-up but were again contacted and interviewed at subsequent follow-up assessments.

Figure 2 - Trajectories of \% total weight loss for P-Group and R-Group Note. P-Group = Group of patients undergoing primary bariatric surgery; R-Group = Group of patients undergoing reoperative surgery; $\% \mathrm{TWL}=$ percentage of total weight loss.

Figure 3 - Trajectories of \% of Maximum Weight Lost for P-Group and R-Group Note. P-Group = Group of patients undergoing primary bariatric surgery; R-Group = Group of patients undergoing reoperative surgery; \% MWL = percentage of maximum weight lost. 